\section{Creating a mosaic in HIV}

\section{By Lauren Martz, Staff Writer}

U.S. researchers have shown that engineered, mosaic HIV vaccines could offer a better in vivo immune response to the virus than vaccines based on natural HIV epitopes. ${ }^{1,2}$ The nearly universal mosaic vaccines trigger a broad cellular immune response, but whether they will also trigger a broad humoral response and viral clearance needs to be tested in humans. An international consortium plans to do just that in a Phase I trial in about a year.

The primary challenge of HIV vaccination is generating an immune response to ever-changing viral epitopes. To complicate matters, HIV epitopes can vary from person to person as well as among geographic regions. And once an individual is infected, the virus can further mutate to avoid the host's immune defenses.

As a result, HIV vaccines must elicit an immune response that recognizes epitopes from a wide swath of viral strains and from mutated variants of particular epitopes.

Previous attempts to create HIV vaccines incorporated antigens based on the sequences of natural HIV peptides. Vaccines based on a natural or consensus sequence of an HIV antigen elicit an immune response against the original epitope and have very little crossreactivity with variants of that epitope.

Such vaccines include Merck \& Co. Inc.'s V520, an adenovirus type 5 (Ad5) vector-based vaccine expressing the natural HIV gag, HIV pol and HIV p27 (nef) antigens. In 2007, Merck discontinued the Phase II STEP trial of V520 after it missed the primary endpoints of preventing HIV infection and reducing viral load. The vaccine triggered a limited $\mathrm{T}$ cell response.

Although Merck hasn't said why it thinks the trial failed, papers from academic groups have suggested that a muted cellular response could have been responsible. The papers suggest that the breadth of epitope-specific $\mathrm{T}$ cell responses directly affects the ability to control HIV replication" ( cine efficacy").

In an effort to expand HIV epitope recognition, in 2006 a team led by Bette Korber at the Los Alamos National Laboratory used an algorithm to generate synthetic peptides composed of nine amino acids representing combinations of all known sequences of a given HIV antigen. The computerized technology was then used to pick the peptide deemed most likely to elicit the broadest immune response. $^{5}$

Now, two papers published in Nature Medicine show that these mosaic vaccines indeed can elicit a broad immune response against HIV epitopes in nonhuman primates. Korber, a laboratory fellow at Los Alamos and an external professor at the Santa Fe Institute, was a principal investigator on both papers.

One study, led by Dan Barouch, chief of the Division of Vaccine Research in the Department of Medicine at the Beth Israel Deaconess Medical Center, tested the breadth of the cellular immune response to mosaic HIV-1 gag, HIV-1 pol and HIV-1 env antigens. They showed that mosaic antigens generated a cellular immune response against a wide array of virus strains compared with natural sequence antigens.

In the second study, Norman Letvin and colleagues obtained similar results using a vaccine consisting of mosaic gag or nef. Letvin is professor of medicine at Beth Israel.

Mark Newman, VP of R\&D at GeoVax Labs Inc., noted that "these mosaic sequences are modified and focused to maximize immunity. By looking at nine amino acids, these peptides could provide great T cell recognition."

GeoVax has an HIV/AIDS vaccine in Phase IIa testing in North and South America. The granulocyte macrophage colony-stimulating factor (GM-CSF; CSF2)-adjuvanted two-component vaccine consists of a recombinant DNA and a recombinant modified vaccinia Ankara (MVA), both of which express gag, pol and env.

The trial is being conducted by the HIV Vaccine Trials Network, which is funded by the NIH's National Institute of Allergy and Infectious Diseases (NIAID).

\section{Good humoral?}

Although the two Nature Medicine studies showed a broad and strong $\mathrm{T}$ cell response, these animal studies couldn't provide data on the extent of the humoral response-which generates antibodies and is responsible for actually clearing infections.

"You can't test HIV antigens in an animal protection model because you can't infect the animals with HIV," noted Shelby O'Connor, associate scientist in the Department of Pathology and Laboratory Medicine at the University of Wisconsin-Madison.

Thus, O'Connor thinks the next step for the Beth Israel group should be to "form mosaic antigens based on SIV antigens, not HIV antigens." Using simian immunodeficiency virus (SIV) would allow animals to be challenged with virus.

Jason Gall, head of the HIV program at GenVec Inc., agreed that preclinical efficacy studies should move to the SIV model. "We want to see an efficacy study for protection" against a repertoire of viral strains, he said. "The strategy of mosaic vaccine development currently focuses on the cellular branch of the immune system. It is still unclear if this is sufficient to provide protection against HIV."

What needs to be considered, added GenVec president and CEO Paul Fischer, is "which part of the immune system to turn on: the humoral or the cellular?"

GenVec's adenoviral HIV vaccine against clades A, B and C, which is being developed with the NIH's Vaccine Research Center, is in 


\section{Box 1. Cellular responses enhance HIV vaccine efficacy.}

In a paper published in Science Translational Medicine, University of Wisconsin-Madison researchers show that eliciting a broad cellular immune response might increase the efficacy of HIV vaccines. ${ }^{6}$ The findings help shore up the hypothesis that broader is better in HIV vaccines and show what has long been suspected: that immune recognition of diverse virus strains improves protection.

Shelby O'Connor and colleagues at the university challenged two types of Mauritian cynomolgus macaques with simian immunodeficiency virus (SIV): animals that were major histocompatibility complex (MHC) heterozygous and those that were MHC homozygous.

MHC class I molecules present viral antigens to $\mathrm{CD}^{+} \mathrm{T}$ cells, and animals heterozygous for the $\mathrm{MHC}$ genes express more diverse variants of the MHC molecules. The diversity translated into improved outcomes. Chronic viremia was 80 times lower in $\mathrm{MHC}$ heterozygous macaques than in $\mathrm{MHC}$ homozygous monkeys.

"What we show in this paper is that certain genetic backgrounds allow some animals to do better than others when challenged with SIV infection. What our finding actually does is suggest that diverse immune responses are favorable for disease outcomes and protect against the infection," said O'Connor, who is an associate scientist in the Department of Pathology and Laboratory Medicine at the university.

The issue is whether the SIV models will translate into humans. "To date, human clinical trials of HIV vaccines have shown little evidence that vac- cination can cause a reduction in viral load or the level of HIV present in the individual following infection," noted Rick King, VP of vaccine discovery at the International AIDS Vaccine Initiative.

O'Connor thinks her team's work addresses this problem and shows that if HIV vaccines can generate a broad cellular response, they will be able to reduce viral load.

She said the findings complement work published in two recent Nature Medicine papers ${ }^{1,2}$ and support the study of polyvalent mosaic antigens in HIV vaccination.

According to O'Connor, a patent has been filed covering the findings in the Science Translational Medicine paper and the IP is available for licensing.

$-L M$

\section{Phase II testing.}

Newman cautioned that producing mosaic SIV vaccines may not be straightforward. "The complication is that SIV variants are often from different monkey species, so mixing the sequences of antigens for this virus might not produce the same results" as mixing HIV antigen sequences, he said.

\section{Time trials}

Rick King, VP of vaccine discovery at the International AIDS Vaccine Initiative, thinks that instead of going into SIV models, the researchers should be developing mosaic vaccines suitable for human trials.

"Only testing in human clinical trials will provide definitive results on whether mosaic antigens will provide control of infection in humans," he told SciBX.

Barton Haynes, a principal investigator on Letvin's paper, said the group plans to do just that. A trial comparing "mosaic envelope immunogens with wild-type and consensus envelopes in a Phase I safety and immunogenicity trial is to start in approximately one year," he told SciBX.

Haynes is director of the Duke Human Vaccine Institute and professor of medicine and immunology at the Duke University School of Medicine. He said the trial will be conducted by the Center for HIV/AIDS Vaccine Immunology, a U.S. university consortium, together with the Bill \& Melinda Gates Foundation, NIAID's Division of AIDS, the HIV Vaccine Trials Network and sanofi-aventis Group's Eurovac/Sanofi-Pasteur S.A. vaccine division.

According to Haynes, Duke, Los Alamos, The University of Alabama at Birmingham and Harvard University have filed for a patent covering the mosaic technology. He said his group plans to offer nonexclusive licenses.
Martz, L. SciBX 3(11); doi:10.1038/scibx.2010.328

Published online March 18, 2010

\section{REFERENCES}

1. Barouch, D. et al. Nat. Med.; published online Feb. 21, 2010; doi:10.1038/nm.2089

Contact: Dan H. Barouch, Beth Israel Deaconess Medical Center, Boston, Mass.

e-mail: dbarouch@bidmc.harvard.edu

2. Santra, S. et al. Nat. Med.; published online Feb. 21, 2010; doi: $10.1038 / \mathrm{nm} .2108$

Contact: Norman L. Letvin, Harvard Medical School, Boston, Mass. e-mail: nletvin@bidmc.harvard.edu

3. Kiepiela, P. et al. Nat. Med. 13, 46-53 (2007)

4. Liu, J. et al. Nature 457, 87-91 (2009)

5. Fischer, W. et al. Nat. Med. 13, 100-106 (2006)

6. O'Connor, S. et al. Sci. Transl. Med.; published online March 10, 2010; doi:10.1126/scitranslmed.3000524

Contact: Dave O'Connor, University of Wisconsin-Madison, Madison, Wis. e-mail: david.h.oconnor@gmail.com

Contact: Shelby O'Connor, same affiliation as above e-mail: shelby.l.oconnor@gmail.com

\section{COMPANIES AND INSTITUTIONS MENTIONED}

Beth Israel Deaconess Medical Center, Boston, Mass. Bill \& Melinda Gates Foundation, Seattle, Wash. Duke University School of Medicine, Durham, N.C. GenVec Inc. (NASDAQ:GNVC), Gaithersburg, Md. GeoVax Labs Inc. (OTCBB:GOVX), Atlanta, Ga. Harvard University, Boston, Mass.

HIV Vaccine Trials Network, Seattle, Wash. International AIDS Vaccine Initiative, New York, N.Y

Los Alamos National Laboratory, Los Alamos, N.M. Merck \& Co. Inc. (NYSE:MRK), Whitehouse Station, N.J.

National Institute of Allergy and Infectious Diseases, Bethesda, Md. National Institutes of Health, Bethesda, Md.

sanofi-aventis Group (Euronext:SAN; NYSE:SNY), Paris, France

Santa Fe Institute, Santa Fe, N.M.

The University of Alabama at Birmingham, Birmingham, Ala. University of Wisconsin-Madison, Madison, Wis. 